\title{
Evolution of the Rdr1 TNL-cluster in roses and other Rosaceous species
}

\author{
Diro Terefe-Ayana, Helgard Kaufmann, Marcus Linde and Thomas Debener*
}

\begin{abstract}
Background: The resistance of plants to pathogens relies on two lines of defense: a basal defense response and a pathogen-specific system, in which resistance (R) genes induce defense reactions after detection of pathogen-associated molecular patterns (PAMPS). In the specific system, a so-called arms race has developed in which the emergence of new races of a pathogen leads to the diversification of plant resistance genes to counteract the pathogens' effect. The mechanism of resistance gene diversification has been elucidated well for short-lived annual species, but data are mostly lacking for long-lived perennial and clonally propagated plants, such as roses. We analyzed the rose black spot resistance gene, Rdr1, in five members of the Rosaceae: Rosa multiflora, Rosa rugosa, Fragaria vesca (strawberry), Malus x domestica (apple) and Prunus persica (peach), and we present the deduced possible mechanism of R-gene diversification.

Results: We sequenced a 340.4-kb region from R. rugosa orthologous to the Rdr1 locus in R. multiflora. Apart from some deletions and rearrangements, the two loci display a high degree of synteny. Additionally, less pronounced synteny is found with an orthologous locus in strawberry but is absent in peach and apple, where genes from the Rdr1 locus are distributed on two different chromosomes. An analysis of 20 TIR-NBS-LRR (TNL) genes obtained from $R$. rugosa and $R$. multiflora revealed illegitimate recombination, gene conversion, unequal crossing over, indels, point mutations and transposable elements as mechanisms of diversification.

A phylogenetic analysis of 53 complete TNL genes from the five Rosaceae species revealed that with the exception of some genes from apple and peach, most of the genes occur in species-specific clusters, indicating that recent TNL gene diversification began prior to the split of Rosa from Fragaria in the Rosoideae and peach from apple in the Spiraeoideae and continued after the split in individual species. Sequence similarity of up to 99\% is obtained between two R. multiflora TNL paralogs, indicating a very recent duplication.

Conclusions: The mechanisms by which TNL genes from perennial Rosaceae diversify are mainly similar to those from annual plant species. However, most TNL genes appear to be of recent origin, likely due to recent duplications, supporting the hypothesis that TNL genes in woody perennials are generally younger than those from annuals. This recent origin might facilitate the development of new resistance specificities, compensating for longer generation times in woody perennials.
\end{abstract}

\section{Background}

Plants are constantly challenged by a large number of different pathogens with diverse infection strategies. To avert these attacks, plants use different mechanisms consisting of active and passive defense lines. Among the active defense mechanisms of plants, specific resistance genes (R-genes) are key factors involved in so-called genefor-gene interactions. Plants harboring a resistance gene

\footnotetext{
* Correspondence: debener@genetik.uni-hannover.de

Institute for Plant Genetics, Leibniz University Hannover, Herrenhaeuser Str. 2, Hannover 30419, Germany
}

recognize specific avirulence (Avr) gene products that characterize particular genotypes of the pathogen [1,2].

Several R-genes have been isolated from a variety of plant species [3]. The majority of R-genes encode nucleotide-binding site (NBS) and leucine-rich repeat (LRR) proteins $[2,4,5]$. On the basis of their $\mathrm{N}$-terminal domains, the NBS-LRR resistance genes can be subdivided into two classes. The first class encodes proteins with an N-terminal TIR domain (homology to the Drosophila Toll and mammalian Interleukin-1 receptors), whereas the second class encodes proteins with coiled-coils (CC), 
sometimes in the form of a leucine zipper (LZ) at the $\mathrm{N}$-terminus of the protein $[3,6,7]$. Two basic strategies for pathogen recognition are currently thought to exist: direct recognition of Avr-gene products by R-proteins and indirect recognition via sensing perturbations of host proteins (the so-called guard hypothesis) [2,8]. Different domains of the NBS-LRR R-genes have been shown to be involved in pathogen recognition, but most studies indicate that the LRR domain plays the most important role in pathogen recognition [9].

Most of the R-genes described to date are organized in clusters reviewed in $[3,10]$. This clustering may facilitate R-gene diversity in the course of adaptation to counteract newly emerging Avr-protein variants in newly evolving virulent races of a pathogen.

Extensive studies have been conducted to understand the mechanism of R-gene diversification, mainly in herbaceous annual plants, such as for $R p 1$ in maize [11-15], $C f 4 / C f 9$ and $M i-1$ in tomato [16-18], $\mathrm{Xa21}$ in rice [19], Dm3 (RGC2) in lettuce [20-23], RPP5 in Arabidopsis [24], $N$ in flax [25] and $R 1$ in potato [26]. Sequence analyses from these studies indicate that R-genes display significantly higher rates of sequence evolution than other plant genes. Furthermore, LRR domains generally evolve more rapidly than the other domains of NBS-LRR genes and often display signs of positive selection. Tandem and segmental gene duplications, recombination, unequal crossing over, point mutations and diversifying selection have been shown to contribute to R-gene diversity. Recent R-gene sequence analyses in Arabidopsis, maize, tomato, barley, lettuce, rice and wheat further indicated illegitimate recombination (IR) as a major source of duplications and deletions [27]. Illegitimate recombination is a type of recombination between two DNA molecules which are not necessarily homologous to each other but share a few identical sequences. These identical sequences are called illegitimate recombination signatures. Illegitimate recombination may result in duplications or deletions [27].

Unlike herbaceous annuals, woody perennial species are characterized not only by long-lived individual plants but also by longer average generation times than annuals. Therefore, the nature of R-gene diversification could vary from that of annual plants. Some perennial plant species, such as roses, propagate clonally as well as sexually via seeds. These different forms of reproduction could also contribute to a possible deviation in R-gene diversity in perennials, as differences in evolutionary rates between annuals and perennials have been noted several times [28]. The mechanisms underlying such differences are still unknown. More frequent and recent duplications of Rgenes have been described in poplar and grapevine compared with rice and Arabidopsis [29], indicating different evolutionary patterns of R-genes between perennial and annual plants.
Roses are attacked by a number of pathogens and pests [30], among which black spot is the most severe disease of field-grown roses. It is caused by the hemibiotrophic ascomycete Diplocarpon rosae, for which a number of pathogenic races have been identified [31]. Resistance to black spot has been found to be caused by both quantitative and qualitative resistance genes [32], with the single dominant R-gene $R d r 1$ from $R$. multiflora being the best studied rose R-gene thus far [33].

Recently, $R d r 1$ was finely mapped to a telomeric position in rose linkage group 1 in a contig of four overlapping BAC clones and isolated via map-based cloning [33-35]. The $R d r 1$ gene is a TIR-NBS-LRR (TNL) type resistance gene and a member of a multigene family of nine highly similar genes clustered in a region of $265.5 \mathrm{~kb}$ in R. multiflora.

Here, we present sequence information from the $R d r 1$ locus of a second rose species, $R$. rugosa, and analyze the sequence conservation of this locus and the TNL family within roses. Furthermore, we analyze synteny with other Rosaceae, represented by sequences from strawberry, peach and apple, and with members of other plant families.

\section{Results}

\section{Comparison of the $R d r 1$ contig between $R$. multiflora and}

\section{R. rugosa}

In addition to the previously published sequence of a 265.5 -kb region spanning the $R d r 1$ locus of $R$. multiflora, a set of four overlapping BAC clones spanning the $R d r 1$ region in $R$. rugosa was sequenced with Roche 454 sequencing. The sequences were assembled to a total length of $340,415 \mathrm{bp}$, with individual sizes of 96.3, 144.9, 75.4 and $78.6 \mathrm{~kb}$ for the BAC clones 31C14, 95G17, 78F5 and 35D6, respectively. The complete sequence has been deposited in GenBank [accession number GenBank: JQ791545]. The first 67,036 bp from the R. rugosa sequence extended beyond the left end of the corresponding $R$. multiflora homologous BAC-clone 2903.

$\mathrm{Ab}$ initio gene prediction revealed 65 protein-coding genes, 46 of which displayed significant similarities to entries in the GenBank database (Table 1). Among the 65 predicted genes, eleven are TNL genes with a high DNA sequence similarity ( $88 \%$ to $95 \%$ ) to the already characterized $R d r 1$ TNL family, and nine are transposable elements (Figure 1). We designated the 11 TNL genes as ruRdr1A through ruRdr1K, where 'ru' stands for the species name of the source of the sequence ( $R$. rugosa), and ' $\mathrm{A}$ ' to ' $\mathrm{K}$ ' indicates the order of the TNL gene in the sequenced contig. A total of 10 of the 11 TNL sequences exhibit uninterrupted predicted coding sequences. We also observed several truncated TNL genes distributed throughout the cluster. Most of these fragments share similarities to LRR domains, and some 
Table 1 List of predicted genes from the 340,415-bp contig of $R$. rugosa orthologous to the Rdr1 locus of $R$. multiflora

\begin{tabular}{|c|c|c|c|}
\hline No. & Position on the contig (bp) & Similarity as revealed by BLASTp (similar to GenBank accession number) & E-value \\
\hline 1 & $6564-11898$ & TIR-NBS-LRR (AEE43932.1) & 0.0 \\
\hline 2 & $12848-11923$ & None & - \\
\hline 3 & $12986-18853$ & Retrotransposon protein, Ty1-copia (ABF96803.1) & 0.0 \\
\hline 4 & $20854-26772$ & Retrotransposon protein, Ty1-copia (ABA98286.2) & 0.0 \\
\hline 5 & 28978-37792 & TIR-NBS-LRR (AEE43932.1) & 0.0 \\
\hline 6 & $41656-37934$ & None & - \\
\hline 7 & $45166-56998$ & Neuroblastoma-amplified sequence (XP_003602296.1) & 0.0 \\
\hline 8 & $60939-57122$ & Major facilitator superfamily domain (XP_003526731.1) & 0.0 \\
\hline 9 & $62610-61549$ & None & - \\
\hline 10 & $62646-64390$ & rhodanese-like domain-containing protein (NP_567785.1) & $1 e^{-102}$ \\
\hline 11 & $68835-64533$ & Vacuolar protein sorting-associated (XP_002274585.1) & 0.0 \\
\hline 12 & 69119-71844 & Transcription factor B3 (ABN06173.1) & $7 e^{-15}$ \\
\hline 13 & 71912-76631 & Gag-pol polyprotein (BAK64102.1) & 0.0 \\
\hline 14 & 76951-79409 & Transcription factor B3 (XP_003517920.1) & $2 e^{-19}$ \\
\hline 15 & $85078-79980$ & TIR-NBS-LRR (AEE43932.1) & 0.0 \\
\hline 16 & $88492-94115$ & None & - \\
\hline 17 & $94486-100495$ & None & - \\
\hline 18 & 104305-100835 & Mutator-like transposase (BAB10320.1) & $1 e^{-102}$ \\
\hline 19 & 109120-104792 & TIR-NBS-LRR (AEE43932.1) & 0.0 \\
\hline 20 & 109935-111490 & None & - \\
\hline 21 & $116498-111549$ & Gag-pol polyprotein (AAO73527.1) & $2 e^{-55}$ \\
\hline 22 & $121170-117613$ & Shikimate dehydrogenase (EEF45470) & $6 e^{-12}$ \\
\hline 23 & $127179-122701$ & TIR-NBS-LRR (AEE43932.1) & 0.0 \\
\hline 24 & 127707-139272 & Non-LTR retroelement reverse transcriptase (AAG13524) & 0.0 \\
\hline 25 & 140854-139893 & None & - \\
\hline 26 & $143524-147207$ & Transcription factor B3 (XP_003535137.1) & $2 e^{-08}$ \\
\hline 27 & 148930-147755 & Phospholipase C (ACF93733.1) & $6 e^{-12}$ \\
\hline 28 & $153630-150256$ & None & - \\
\hline 29 & $161422-157057$ & TIR-NBS-LRR (AEE43932.1) & 0.0 \\
\hline 30 & $166328-163477$ & LRR (AEE43932.2) & $4 e^{-85}$ \\
\hline 31 & $169607-170212$ & None & - \\
\hline 32 & $176508-171812$ & TIR-NBS-LRR (AEE43932.1) & 0.0 \\
\hline 33 & $176830-179287$ & Transcription factor B3 (XP_003517920.1) & $2 e^{-21}$ \\
\hline 34 & 179594-182362 & Transcription factor B3 (XP_003517920.1) & $3 e^{-18}$ \\
\hline 35 & $188265-182622$ & TIR-NBS-LRR (AEE43932.1) & 0.0 \\
\hline 36 & 198290-192614 & TIR-NBS-LRR (AEE43932.1) & 0.0 \\
\hline 37 & 199678-207935 & None & - \\
\hline 38 & 211387-209495 & None & - \\
\hline 39 & $217944-212928$ & TIR-NBS-LRR (AEE43932.1) & 0.0 \\
\hline 40 & $230339-218090$ & Retrotransposon protein, Ty1-copia (ABF96803.1) & 0.0 \\
\hline 41 & 232191-238449 & Non-LTR retroelement reverse transcriptase (AAB82639) & 0.0 \\
\hline 42 & $239180-238929$ & None & - \\
\hline 43 & $239877-240955$ & None & - \\
\hline 44 & $245771-241137$ & TIR-NBS-LRR (AEE43932.1) & 0.0 \\
\hline
\end{tabular}


Table 1 List of predicted genes from the 340,415 -bp contig of $R$. rugosa orthologous to the Rdr1 locus of $R$. multiflora (Continued)

\begin{tabular}{|c|c|c|c|}
\hline 45 & $250194-245924$ & Copia-type polyprotein (AAG51247.1) & 0.0 \\
\hline 46 & $250788-253960$ & None & - \\
\hline 47 & $254886-254010$ & None & - \\
\hline 48 & $258861-257151$ & ATP binding protein (XP_002515676.1) & 0.0 \\
\hline 49 & $265170-261078$ & AAA domain-containing protein (XP_003544721.1) & 0.0 \\
\hline 50 & $266721-274192$ & Yellow stripe-like protein (XP_003602315.1) & 0.0 \\
\hline 51 & $278098-274211$ & GTPase-activating protein (XP_003526739.1) & 0.0 \\
\hline 52 & 283722-281796 & 6-phosphogluconolactonase (XP_002518214.1) & $5 e^{-135}$ \\
\hline 53 & $285094-286783$ & Ubiquitin (XP_002530306.1) & $4 e^{-88}$ \\
\hline 54 & 288959-286931 & Aldo-keto reductase (XP_003602320.1) & 0.0 \\
\hline 55 & 290639-289124 & Homeobox leucine zipper protein (AAD38144.1) & $1 e^{-79}$ \\
\hline 56 & 298129-297002 & None & - \\
\hline 57 & 298247-299808 & Hypothetical protein (XP_003602325.1) & $5 e^{-97}$ \\
\hline 58 & $309056-299987$ & TOPLESS-RELATED protein (XP_002275116.1) & $4 e^{-105}$ \\
\hline 59 & 309339-311499 & None & - \\
\hline 60 & $318648-317787$ & None & - \\
\hline 61 & $325145-319916$ & Serine/threonine protein kinase (NP_001234146.1) & 0.0 \\
\hline 62 & $326294-328526$ & None & - \\
\hline 63 & $331756-329732$ & UDP-N-acetylglucosamine transporter (XP_003531350.1) & $5 e^{-48}$ \\
\hline 64 & $335561-332977$ & F-box protein (XP_003610959.1) & $2 e^{-08}$ \\
\hline 65 & $336756-340415$ & Unnamed protein product (CBI23069.3) & 0.0 \\
\hline
\end{tabular}

Gene predictions were made using FGENESH and BLAST.

are attached to retroelements. Six of the transposable elements belong to the group of long terminal repeat (LTR) retrotransposons; two belong to the non-long terminal repeats (non-LTRs) retrotransposons; and one belongs to the mutator-like transposase type of DNA transposons.

Similar to the previously described $R$. multiflora TNLs [35], the size of the genomic sequences of the $R$. rugosa TNLs varies from $4329 \mathrm{bp}$ to $5677 \mathrm{bp}$. The ruRdr1B TNL carries an additional 1904-bp sequence of unknown function in the third intron (Figure 2). Most of the TNL homologs are comprised of four exons and three introns with few homologs having five exons that correspond to the TIR, NBS, NLL (NBS-LRR linker), LRR and post-LRR domains, respectively (Figure 2).

The additional sequence that extends the $R$. rugosa contig beyond the borders of the $R$. multiflora contig contains two TNL elements (ruRdr1A and ruRdr1B) as well as two transposable elements and sequences with similarity to a neuroblastoma amplified gene, a major facilitator superfamily domain and a rhodanese-like domain-containing protein.

GATA alignment and dot plot comparison of the $265.5-\mathrm{kb}$ region from $R$. multiflora and the $340.4-\mathrm{kb}$ region from $R$. rugosa indicates a high degree of synteny between the two species (Figure 1).
A group of nine sequences (ATP binding, AAA type ATPase, Yellow stripe-like, GTPase activator, 6phosphogluconolactonase, Ubiquitin fusion, Homeobox leucine zipper, TOPLESS-RELATED and Serine/threonine protein kinase) at the right end of the $R$. multiflora contig and a sequence stretch comprising a predicted gene for a vacuolar protein sorting-associated protein and transcription factor at the left end of the contig are perfectly conserved between the two species. However, the region between these sequences exhibits several copy number changes, inversions and deletions/ insertions. Of the $11 \mathrm{TNL}$ genes located on the $R$. rugosa contig, nine are in the same and two are in a reverse orientation compared with the $R$. multiflora contig in which all of the TNL genes are in the same orientation. For some of the non-TNL genes, differences are observed in terms of the relative location and the number of homologs within each cluster. Furthermore, some sequences are completely missing in one cluster and present in another. For example, a 23-kb region with similarity to prolyl 4-hydroxylase alpha, aminotransferase-like, WUSCHEL protein terminator and inosine-5'-monophosphate dehydrogenase is present in the homologous locus in $R$. multiflora but absent in the R. rugosa locus. 

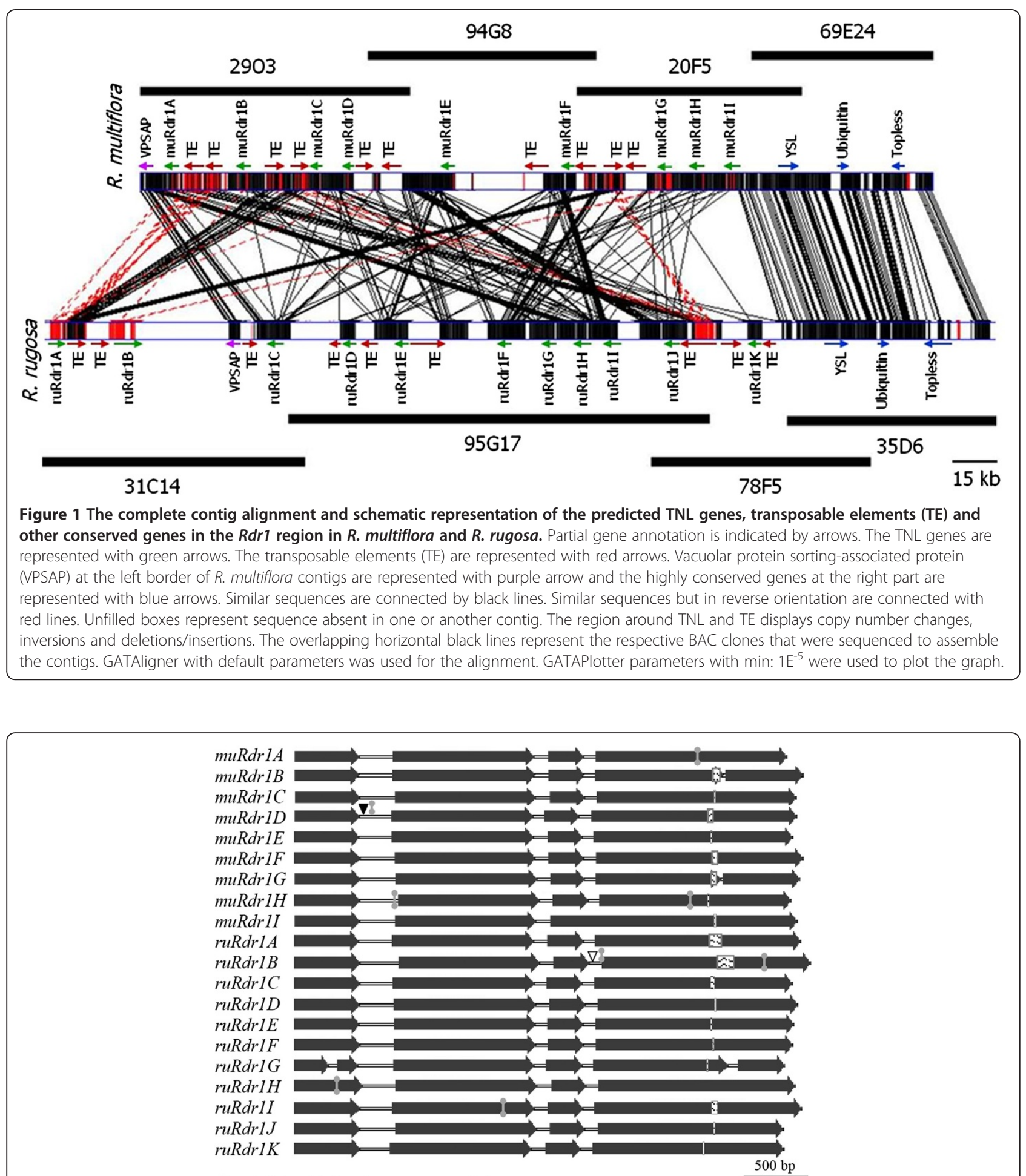

Indels with IR signatures $\quad$ Hypervariable region with $(\mathrm{CT})_{\mathrm{n}} \overline{\text { repeat }}$

$\nabla$ 1904-bp insertion of unknown function with IR signatures

$\boldsymbol{\nabla}$ 6957-bp transposable element insertion with IR signatures

Figure 2 The intron-exon structures of the $\mathbf{2 0} R d r 1$ homologs from roses. The majority of the $R d r 1$ genes include four exons and three introns with few homologs having five exons that correspond to the TIR, NBS, NLL (NBS-LRR linker), LRR and post-LRR domains, respectively. Several illegitimate recombination (IR) signatures are distributed across the sequence, flanking indels or repeats. The LRR region is characterized by variable number of $(C T)_{n}$ repeats ranging from zero in muRdr1A and ruRdr1H to 65 in ruRdr1B [41]. 
In addition, the transposable elements distributed over the two contigs differ both in their position and sequence. The ten transposable elements in the $R$. multiflora locus belong to the Ty1/copia type retroelements, whereas the $R$. rugosa locus contains Ty1/copia as well as other different retroelements.

\section{Conservation of the locus within the Rosaceae}

Among the plant species with sequenced genomes, the strawberry is the closest relative to the genus Rosa. We therefore compared the $R$. multiflora contig to sequences from Fragaria vesca. We subjected individual sequences from the $R d r 1$ contig to BLAST searches against the Fragaria genome sequence and located a stretch of similar sequences of approximately $354 \mathrm{~kb}$ from strawberry chromosome 7 between positions $19,798,478$ bp and $20,152,477$ bp. Several insertions, deletions and large rearrangements in the form of inversions and translocations were found (Additional file 1). The cluster of conserved genes from the right and the left sides of the $R$. multiflora contig are also found in Fragaria. Twelve TNL genes with high similarity to the $R d r 1$ gene family (71\% to $87 \%$ identity at the DNA level and $61 \%$ to $81 \%$ similarity at the amino acid level) are also located in the selected Fragaria sequence region. In the following analyses, we designate these genes as FvTNL1 through FvTNL12. Unlike at the $R$. multiflora locus, the orientation of the Fragaria TNLs varies, in that the majority of the genes are inverted relative to the $R$. multiflora copies. Further differing from the $R$. multiflora locus, the genes within the conserved cluster from the right side of the contig, such as the yellow stripe-like gene and the ubiquitin fusion gene, are in an inverted position, inserted within the TNL genes.

The 354-kb Fragaria sequence contains a stretch of $14.2 \mathrm{~kb}$ of ambiguous sequence (represented by stretches of Ns) resulting from problems in the assembly. This may lead to changes in the Fragaria locus structure in the future, although this is unlikely.

In contrast to what is observed in Fragaria, the $R d r 1$ homologous locus is located on two different chromosomes in P. persica (Additional file 2). The closest relatives to the $R d r 1$ gene family are found in a cluster of 15 genes in linkage group 8 (scaffold no. 8 from bases $2,050,000 \mathrm{bp}$ to $2,510,000 \mathrm{bp}$ ), whereas the genes flanking the TNL cluster at the right and left margins in Rosa and Fragaria are located in linkage group 2 (scaffold no. 2 from 26,050,000 bp to 26,110,000 bp) in Prunus. The 15 Prunus TNLs are designated PpTNL1 through PpTNL15. The cluster is characterized by large differences in terms of the non-TNL genes and the orientation of the TNLs. In contrast, the flanking genes are highly conserved between Rosa and Prunus.

The similarity between rose and apple is comparable to the above-mentioned situation in peach, in which
TNLs and flanking genes are located in two different linkage groups (Additional file 3). The closest relatives to the Rdr1 TNLs are located in a cluster of 11 genes in apple linkage group 15 spanning a position from $41,166,396 \mathrm{bp}$ to $41,719,891 \mathrm{bp}$. Hereafter, they are designated MdTNL1 through MdTNL11. The flanking genes are located in linkage group 1 (position 35,200,000 bp to $35,294,999 \mathrm{bp}$ ).

\section{Conservation of the locus in taxa outside of the Rosaceae}

The strong synteny of the Rdr1 locus between Rosa and Fragaria and the low synteny with Prunus and Malus raise the question of whether the group of $R d r 1 \mathrm{TNL}$ genes is present at a similar locus in other plant families. We defined synteny simply as close linkage of a TNL cluster with similarity to the $R d r 1$ family to the flanking genes conserved among the Rosoideae species.

The first species investigated was Medicago truncatula, as the Fabaceae are a family closely related to the Rosaceae. We found some of the flanking genes of the right side of the contig distributed among more than three chromosomes in M. truncatula, but no TNL genes related to the $R d r 1$ family are located close to that locus (Additional file 4). We also did not detect the vacuolar protein sorting-associated protein or transcription factor from the left side of the $R$. multiflora locus, indicating a lack of synteny in Medicago. Related TNL genes are found on several of the Medicago chromosomes, but the similarity to the $R d r 1$ family is too low to infer orthology relationships. The $M$. truncatula sequences utilized in these analyses are downloaded from http:// www.medicagohapmap.org/.

We performed the same analysis in the Arabidopsis thaliana genome, and again, no syntenic block of sequences could be detected (Additional file 5). The flanking genes in this case also do not form a cluster at one location but are distributed on more than two chromosomes in more than two copies. The A. thaliana sequences utilized in these analyses are downloaded from http://www.arabidopsis.org/.

\section{Evolutionary history of the Rdr1 TNL family}

On the basis of our first analyses of the conservation of the structure of the $R d r 1$ locus, we performed a phylogenetic analysis of the TNL genes from the $R$. multiflora, $R$. rugosa and $F$. vesca contigs. In addition, we included all 15 peach and 11 apple genes clustered in linkage groups 8 and 15, respectively. Overall, the derived amino acid sequences of 53 full-length TNL genes were aligned (three truncated genes from apple and two from Fragaria were discarded because they did not contain TIR, NBS and LRR domains), and a maximum likelihood phylogenetic tree was constructed with 500 bootstrap replicates (Figure 3). 


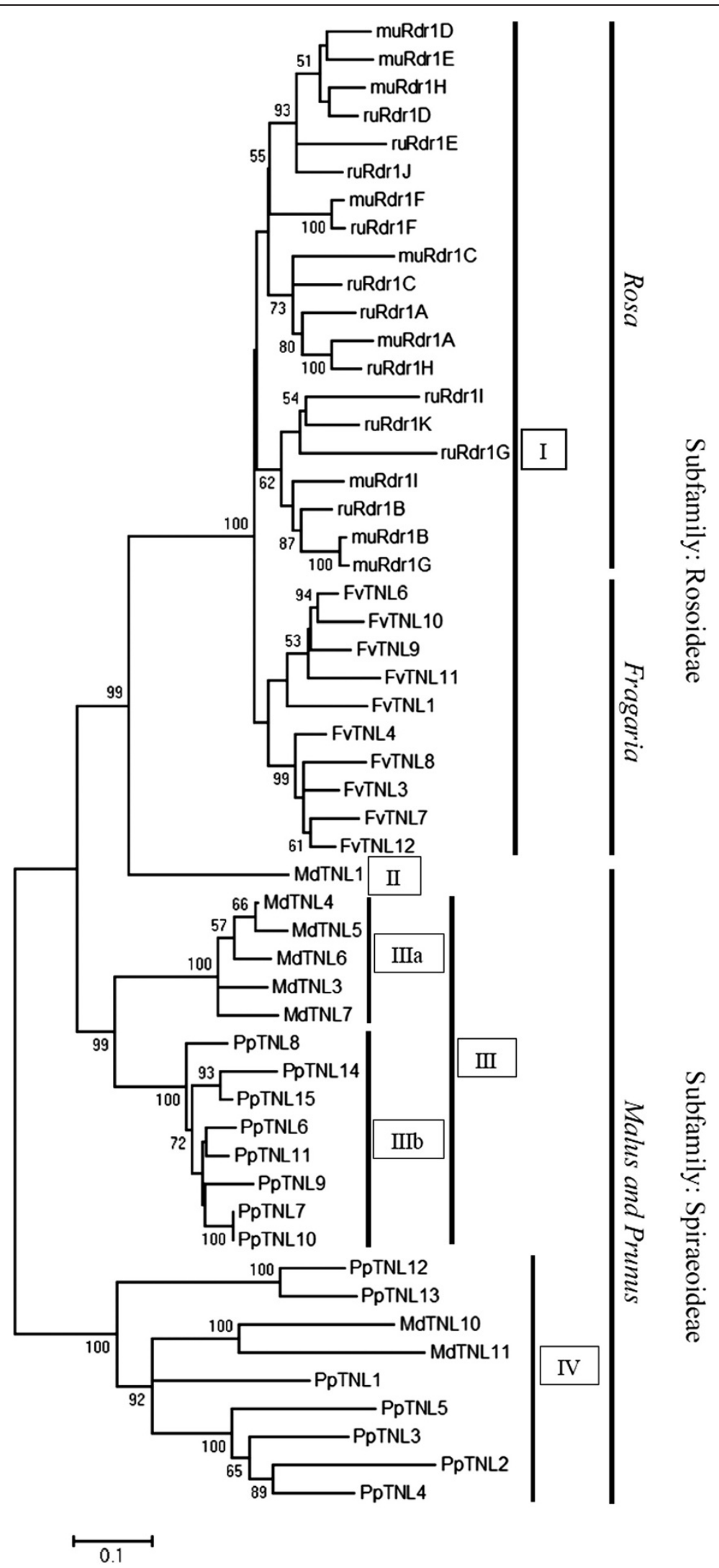

Figure $\mathbf{3}$ (See legend on next page.) 
(See figure on previous page.)

Figure 3 Phylogenetic analysis of the amino acid sequences of the 53 TNLs. The evolutionary history was inferred using the Maximum Likelihood method based on the JTT matrix-based model. The bootstrap consensus tree inferred from 500 replicates is taken to represent the evolutionary history of the sequences analyzed. Branches corresponding to partitions reproduced in less than $50 \%$ of bootstrap replicates are collapsed. The two subfamilies, Rosoideae and Spiraeoideae, are clearly separated. The TNLs from the genera Rosa and Fragaria are also separate. Intermixed branching is shown for the 20 Rdr1-TNLs from R. multiflora and R. rugosa. The 23 TNLs of apple and peach are clustered in multiple clades or subclades. The TNLs for each clade or subclade are species specific, with few intermixing. The tree is drawn to scale, with branch lengths measured in terms of the number of substitutions per site. Bootstrap values of more than 50 are indicated next to the branches.

The tree shows four major groups that are highly supported by bootstrap values of $99 \%$ to $100 \%$. There is a distinct cluster (I) formed by Rosa and Fragaria, representing the subfamily Rosoideae of the Rosaceae, which is separated from a single Malus sequence (MdTNL1, II), a cluster (III) in which sequences from Malus and Prunus each form distinct subclusters and a cluster (IV) comprised mainly of Prunus sequences and two Malus sequences in one subcluster (Figure 3). Within the largest Rosoideae cluster, sequences from Rosa and Fragaria each form distinct highly supported subclusters, indicating recent evolution of the Rdr1 TNLs after the genera diverged. Within the subcluster comprised of the rose sequences, there is no separation of $R$. multiflora and $R$. rugosa sequences. Instead, highly supported clusters with pairs of sequences from both species (e.g., muRdr1F/ruRdr1F, muRdr1A/ruRdr1H) and mixed subclusters with low bootstrap support indicate that some of the sequences evolved before the species separated. As an exception to this, the pair muRdr1B/muRdr1G shows almost no divergence, indicating a recent gene duplication in Rosa multiflora.

In contrast to the divergence of sequences from Rosa and Fragaria, the Rdr1 homologues in Prunus and Malus are mixed in clusters III and IV. Cluster III is comprised of two subclusters (IIIa and IIIb), each including genes only from Prunus or Malus, in contrast to cluster IV, which consists of genes from both species. The branch lengths of subclusters IIIa and IIIb are shorter than the branch lengths of cluster IV, indicating that they evolved after Prunus and Malus diverged.

\section{Sequence evolution of the TNL family}

To obtain additional information about the processes that led to the diversity of the Rdr1 TNL genes, we analyzed the sequence variability of the genes in more detail.

Analyses of nucleotide diversity $(\pi)$ among the TNL genes from Rosa ranged from 0.0046 between the closely related paralogs muRdr1B and muRdrlG (DNA identity 99\%) to 0.1413 between muRdr1F and ruRdr1G (Figure 4).

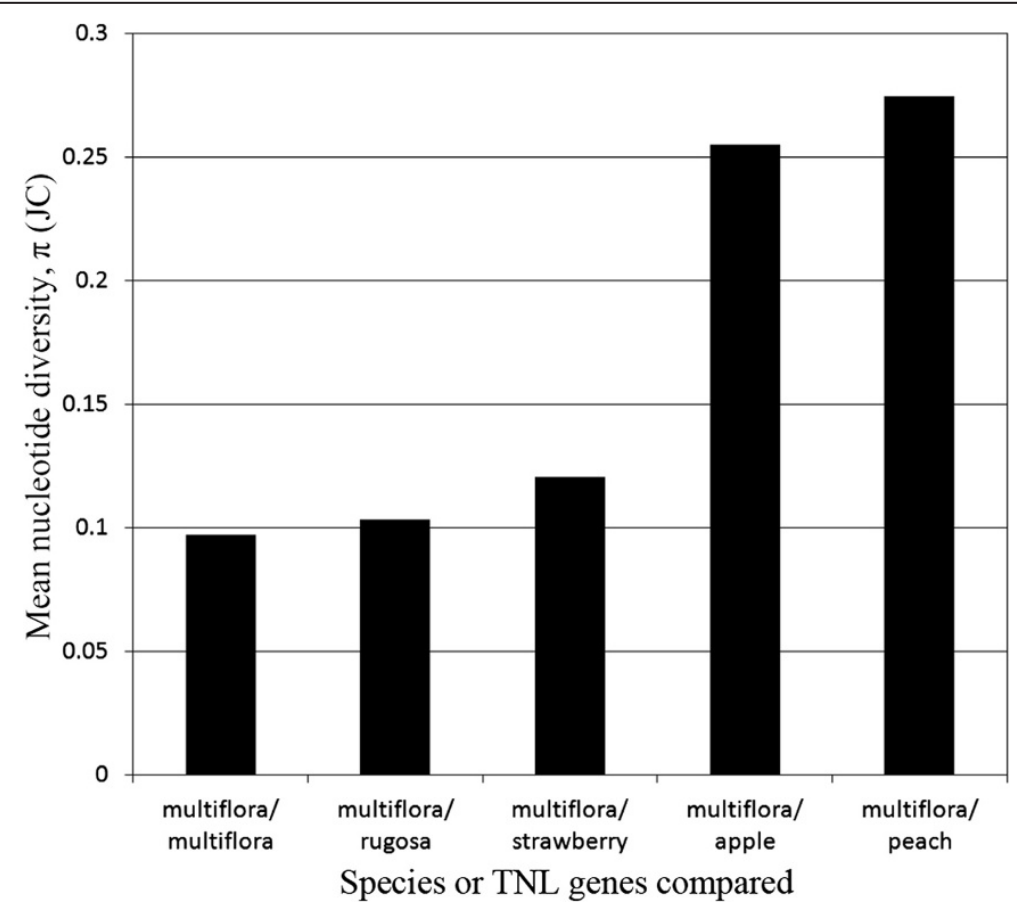

Figure 4 Average nucleotide diversity values $(\pi)$ within $R$. multiflora Rdr1-TNL genes and between R. multiflora and four other species of the Rosaceae. 
The nucleotide diversity within the cluster of $R$. multiflora TNL genes is at approximately the same level as the average for the $R$. multiflora genes compared with the genes from $R$. rugosa. Surprisingly, comparison of all $R$. multiflora TNL genes with the genes from the $F$. vesca cluster leads to only slightly higher values (all approximately 0.1 ) than comparisons among the rose genes. In contrast, a comparison with the other two rosaceous genomes (Malus and Prunus) leads to a sharp increase in the diversity, associated with an average $\pi$ value of 0.2492 (Figure 4). If TNL and non-TNL genes from the orthologous regions are compared, the value is much higher than the average value for the conserved cluster of flanking genes $(\pi=0.1)$ or the value for a randomly selected single-copy control gene (AGT, alanine:glyoxylate aminotransferase) from an EST collection $(\pi=0.099)$. This indicates higher evolutionary rates for the TNL genes compared with other genes from the same locus or unlinked loci (Figure 5). The large variation in sequence similarity between the 53 Rosaceae TNLs obscured the obvious difference in diversity and led to a similar level of nucleotide diversity for the TIR, NBS and LRR domains (Figure 5). A clear difference is observed between the TIR, NBS and LRR domains when the more similar TNL genes from $R$. multiflora, $R$. rugosa and /or Fragaria are compared (Additional file 6), which is in line with previous reports in which the TIR domain is the most conserved region, and the NBS and LRR domain regions are more diverse.

As several studies on the evolution of TNL genes in plants have indicated positive selection acting on the LRR region, we computed $\mathrm{Ka}$ and $\mathrm{Ks}$ values for the whole coding region of all 20 rose TNL genes. The overall mean Ks values estimated using the modified Nei and
Gojobori algorithm [36] with Jukes-Cantor correction [37] are $0.12,0.150$ and 0.11 for the TIR, NBS and LRR domains, respectively. Whereas the overall mean Ka estimates are 0.06, 0.10 and 0.08 for the TIR, NBS and LRR domains, respectively. Recalculation over short sequences by dividing each of the domains into two to four parts revealed elevated Ka over Ks values for a few pairwise comparisons, particularly in the LRR region, which is indicative of positive selection (Figure 6). The number of comparisons with an elevated Ka over Ks increases from the TIR to NBS domains, and the highest numbers occurred in the LRR domain region (Figure 6). This is in agreement with several previous studies, in which different ratios of Ka to Ks were found for the different subunits of the LRR domains, with the highest ratio of Ka to Ks observed for the solvent-exposed residues of the LRR subunit, which are speculated to determine resistance specificity $[16,38]$. The smallest Ks and Ka values are 0.012 and 0.005 , respectively, detected between the most similar $R d r 1$ paralogs, $m u R d r 1 B$ and muRdr1G.

Comparison of the $20 R$. multiflora and $R$. rugosa TNLs revealed several duplications and deletions distributed along the entire domain of the gene. Similar to previous observations related to $\mathrm{R}$-gene sequences in annual plant species [27], illegitimate recombination (IR) signatures are detected flanking eight duplications and indels (Figure 2). For example, an 'AAT' fingerprint flanked the 27-bp repeat in the TIR-encoding domain of ruRdr1H; a 'CAGAG' fingerprint flanked the 18-bp repeat in the NBS-encoding domain of $m u R d r 1 H$; and an 'AC' fingerprint flanked the 16-bp repeat in the LRR-encoding domain of muRdr1A (Figure 7). In most cases, the IR signatures are $100 \%$ identical.

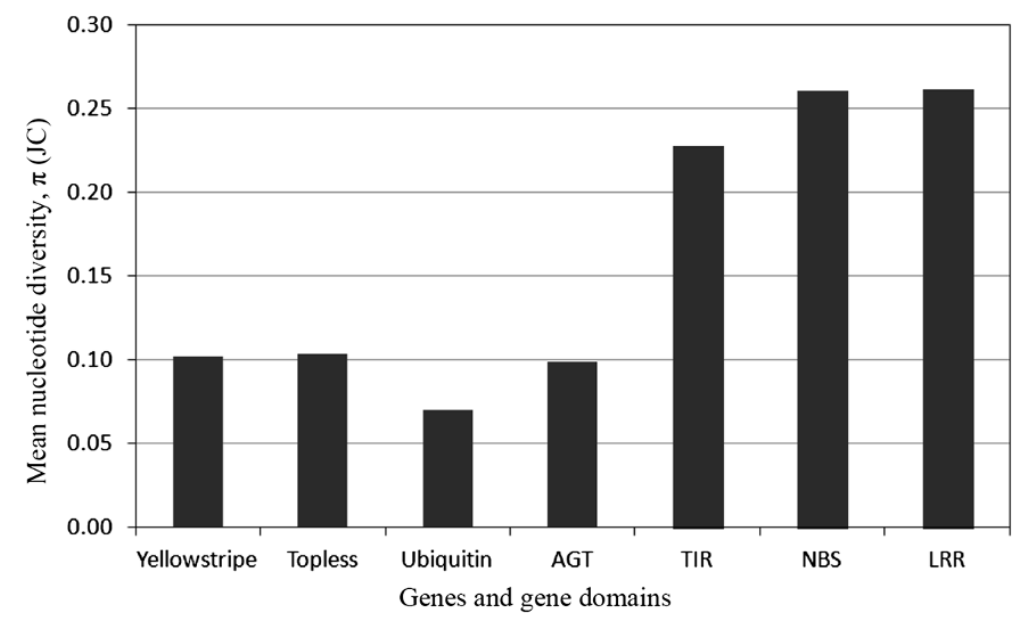

Figure 5 Average nucleotide diversity between the 53 Rosaceae Rdr1-TNLs and between the three flanking genes as well as the AGT gene. The Rosaceae TNLs and their domains are characterized by larger mean $\pi$ values than the flanking genes and AGT (alanine:glyoxylate aminotransferase). AGT is present as an arbitrarily selected gene, which occurs as a single copy gene in most plant genomes. 


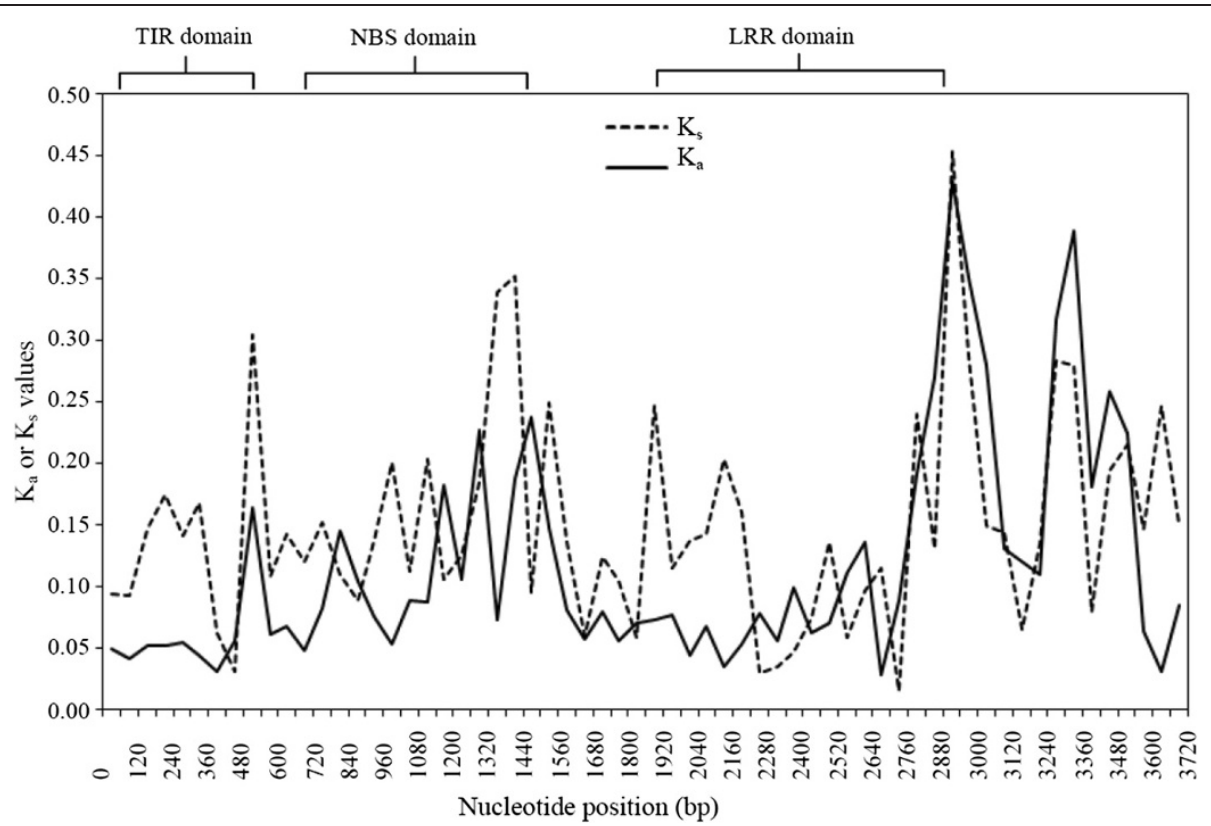

Figure 6 Sliding window analyses of synonymous and nonsynonymous substitutions in different regions of the 20 Rdr1-TNLs sequences from $\boldsymbol{R}$. multiflora and $\boldsymbol{R}$. rugosa. Low Ka/Ks ratios $(<1.0)$ dominate the entire sequence, but few regions with elevated Ka/Ks ratios $(>1.0)$ can be found, increasing in number towards the LRR region.

Approximately 1270 polymorphic sites and 87 indels varying in length from $1 \mathrm{bp}$ to $160 \mathrm{bp}$ are observed. The longest indel is in the microsatellite repeat region in exon 4. Some indels are duplications flanked by IR signatures resulting from unequal crossing over. The majority of indels are in the noncoding region of the TNL sequences.

Analyses with Geneconv [39] uncovered 26 gene conversion tracts among the 20 TNLs of $R$. multiflora and R. rugosa (Table 2). Tracts of $100 \%$ identical sequences are identified as an indication of sequence conversion. Sequence conversion events were visually confirmed in different regions of the TNL genes.

\section{Discussion}

\section{The Rdr1 locus is conserved within the Rosa genus}

We sequenced a genomic region of $340.4 \mathrm{~kb}$ from $R$. rugosa orthologous to the recently published $R d r 1$ gene cluster from $R$. multiflora. Comparison of the two regions reveals a high degree of conservation of genes flanking a cluster of 11 TNL genes, but we also found rearrangements within the group of TNLs. This corresponds to other studies in which major rearrangements, including deletions, gene duplications and inversions, have been found among groups of NBS-LRR genes [7]. The structure of the $R d r 1$ locus indicates several mechanisms that have led to the above-mentioned structural differences between the two orthologous regions. The close relationships between the TNL genes, presenting DNA similarities with between an 88\% and 95\% identity, might have led to unequal crossing over, resulting in some of the observed duplications/deletions. Another factor promoting recombination at the $R d r 1$ locus is the presence of transposable elements belonging to the Ty1/Copia class as well as non-LTR classes, which are present on both contigs. Several authors have hypothesized that both the repetitive nature of various copies of retroposons and their capacity to transpose neighboring genes have likely contributed to the diversity of R-gene clusters [10,40]. The high variability detected among the TNL genes at the locus is also reflected in the large number of alleles for a microsatellite from the LRR region of the $R d r 1$ gene family that has been analyzed in rose varieties, species and individuals from natural populations of the diploid species Rosa arvensis [41].

\section{The Rdr1 locus is conserved between Rosa and Fragaria}

Comparing the rose $R d r 1$ locus with a syntenic region of the Fragaria genome revealed conservation of the flanking genes as well as the presence of the Rdr1 TNL gene family. This finding is in agreement with synteny studies conducted with molecular markers showing that the region on rose chromosome 1 to which we mapped $R d r 1$ is syntenic to Fragaria chromosome 7 [42,43]. It also indicates that a cluster of TNL genes with similarity to the Rdr1 TNLs existed prior to the separation of the tribe Rosa (to which roses belong) from the Potentilleae 


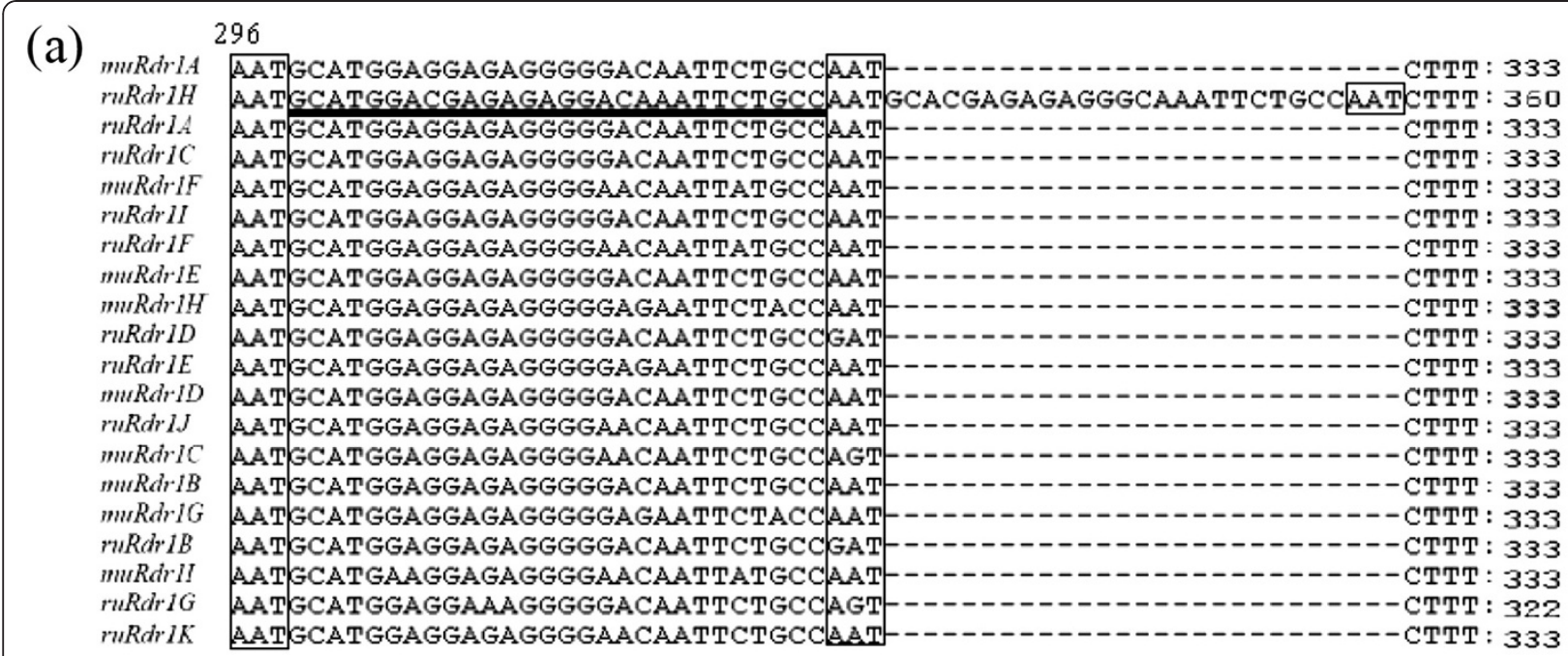

(b) 750

miRdrlA TCTTGTTGTAGGTACGA_A.A CACAG- ruRdrlH TCTTGTTGTAGGTACGAAA CACAG--

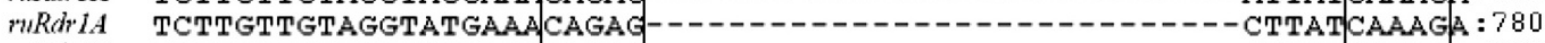

ruRdrlC TCTTGTTGTAGGTACGAAA CACAG----n

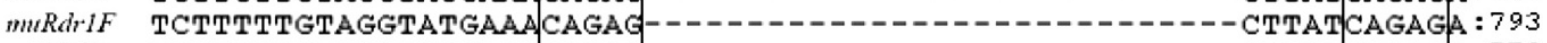

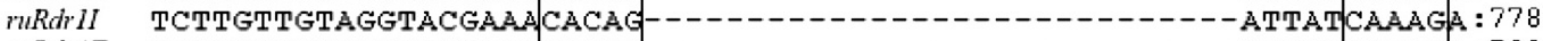

MRdrIF TCTTTTTGTAGGTATGAAA|CAGAG-

$m$ mRdrlE TCTTATTGTAGGTATGAAACTGAG---nA

$m u R d r l H$ TCTTATTGTAGGTATGAAA CAGAGCTTATTCTATTGTAGGTATGAAACAGAGCTTATCAGAGA: 821

ruRdr1D TCTTGTTGTAGGTACGAAA|CACAG - 10

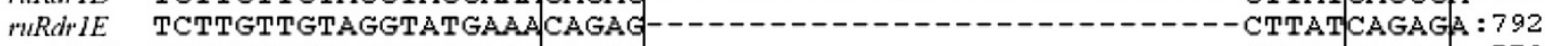

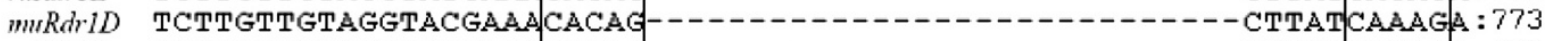

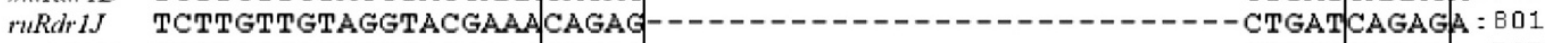

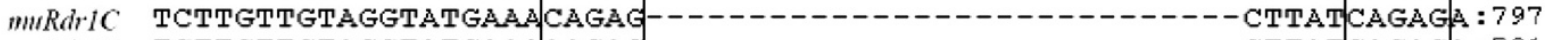

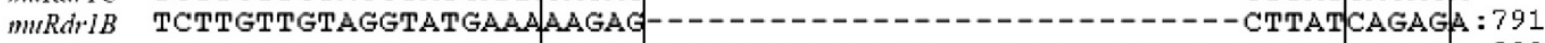

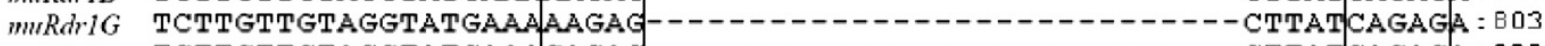

ruRdr1B TCTTGTTGTAGGTATGAAA CAGAG--

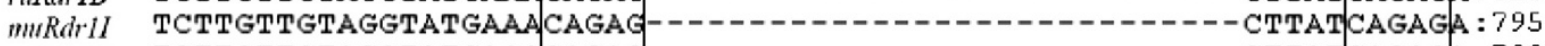

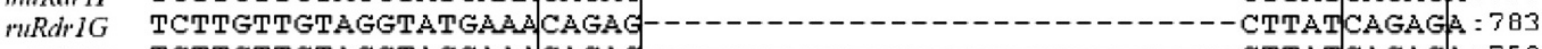

(c)
$r u R d r I K$
TCTTGTTGTAGGTACGAAACACAG

muRdrlA 3110 : ACAAGTGTTRCCAGATCOACAAGTGTTTCCAGAACCACCAAA: 3151

$m u R d r I F 3111$ : ACAAGTGTTTCCAGATCC-------------- ACCAAA: 3134

ruRdr1I 3106: ACAAGTGTTTCCAGATCC---------------ACCAAA: 3129

niRdr1J 3126: ACAAGTGTTTCCAGATCC--------------ACCAGA: 3149

muRdrIC $3120:$ ACAAGTGTTTCCAGATCC---------------ACCAGA: 3143

Figure 7 Illegitimate recombination (IR) signatures flanking different regions of the 20 Rdr1-TNL homologs. a) IR-flanking TIR domain. b) IR-flanking NBS domain. c) IR-flanking LRR domain. Two to five base IR signatures are framed. The sequence region with random duplication is underlined. Certain sequence parts of the IR signatures are imperfect with few mismatches. In each case, duplication of at least one sequence is visible following the IR signature.

(to which strawberries belong). Apart from the flanking genes and the presence of TNLs with high similarity to the $R d r 1$ family, large structural changes occurred, in that many non-TNL genes found in rose do not match similar genes in strawberry and vice versa. As the two species belong to separate tribes, this is not surprising, given the variability that we detected between the two rose species. The mechanisms leading to these differences are likely the same mechanisms that shaped the differences within the genus Rosa.
The Rdr1 locus is not conserved between Rosa, Prunus and Malus

In contrast to the conservation of the $R d r 1$ locus in Fragaria, no conservation of the TNL cluster is found in Malus and Prunus. Although the flanking genes are conserved as a tightly linked group in both Malus and Prunus, there are no closely linked TNLs, as observed in Fragaria. Rather, the closest relatives to the rose $R d r 1$ family are detected in clusters on separate chromosomes. As conservation is also lacking in Medicago and Arabidopsis, one 
Table 2 The $\mathbf{2 6}$ gene conversion tracts detected in the $\mathbf{2 0}$ TNL homologs of the two rose species, R. multiflora and R. rugosa

\begin{tabular}{|c|c|c|c|}
\hline TNLs & $\begin{array}{c}\text { Sequence } \\
\text { tract begin (bp) }\end{array}$ & $\begin{array}{c}\text { Sequence } \\
\text { tract end (bp) }\end{array}$ & $\begin{array}{l}\text { Sequence } \\
\text { length (bp) }\end{array}$ \\
\hline ruRdr1H;ruRdr1I & 722 & 956 & 235 \\
\hline muRdr1F;ruRdr1F & 1515 & 1800 & 286 \\
\hline ruRdr1H;ruRdriJ & 1580 & 1701 & 122 \\
\hline muRdr1F;ruRdr1F & 1127 & 1391 & 265 \\
\hline ruRdr1A;ruRdriJ & 1580 & 1715 & 136 \\
\hline muRdr1F;ruRdr1I & 2868 & 3125 & 258 \\
\hline ruRdr1H;muRdriC & 2664 & 2843 & 180 \\
\hline muRdr1H;muRdr1B & 1 & 209 & 209 \\
\hline ruRdr1H;ruRdr1A & 1552 & 1701 & 150 \\
\hline muRdr1C;ruRdr1K & 2823 & 2995 & 173 \\
\hline muRdr1H;muRdr1G & 1 & 205 & 205 \\
\hline ruRdr1l;ruRdr1J & 2243 & 2394 & 152 \\
\hline ruRdr1H;ruRdr1G & 3111 & 3227 & 117 \\
\hline muRdr1F;ruRdr1F & 2001 & 2333 & 333 \\
\hline ruRdr1 J;ruRdrlK & 229 & 445 & 217 \\
\hline muRdr1H;muRdr1G & 211 & 418 & 208 \\
\hline muRdr1F;ruRdr1F & 182 & 519 & 338 \\
\hline muRdr1F;ruRdr1I & 2651 & 2866 & 216 \\
\hline ruRdr1A;ruRdr1C & 211 & 428 & 218 \\
\hline muRdr1A;muRdr1I & 535 & 624 & 90 \\
\hline ruRdr1C;ruRdr1I & 276 & 453 & 178 \\
\hline muRdr1A;muRdr1D & 233 & 416 & 184 \\
\hline muRdr1D;ruRdr1J & 1704 & 1839 & 136 \\
\hline ruRdr1l;ruRdr1K & 1598 & 1676 & 79 \\
\hline muRdr1F;ruRdr1I & 3992 & 4179 & 188 \\
\hline muRdr1l;ruRdriG & 1717 & 1841 & 125 \\
\hline
\end{tabular}

possible conclusion is that the TNLs were inserted at their present location after the Rosoideae and Spiraeoideae split. Although errors during whole genome sequencing and assembly cannot be excluded [44], the cause of this transposition is difficult to infer. However, the presence of mobile genetic elements is one possible explanation. Comparative analysis of an NBS-LRR cluster in Phaseolus vulgaris and other Fabaceae indicates that subtelomeric positions are prone to transpositions of repeated DNA elements [45]. This might be the case for the $R d r 1$ locus split in Spiraeoideae, as the Rdr1 TNL clusters are located at a telomeric position in the rose chromosome 1 map [33]. In contrast to the situation in Phaseolus, we have no evidence that any sequence closely related to the $R d r 1$ family maps outside the cluster on linkage group 1. Mapping experiments in two diploid and one tetraploid mapping population reveal that all polymorphic fragments are linked in one region on linkage group 1, although they spread over a distance of up to $18 \mathrm{cM}(42)$.

\section{Phylogenetic analyses}

Phylogenetic analyses (Figure 3) led to a dendrogram that was highly supported by bootstrap values above 80\%, in which the rose and Fragaria genes form separate clusters, whereas the Malus and Prunus genes are located in mixed clusters. None of the Fragaria genes clustered with any of the rose genes, which form clusters according to the two different rose species.

This indicates that although the TNL genes most likely translocated to their current positions after the separation of the Rosoideae and the Spiraeoideae, the locus underwent further independent evolution in each of the genera. This is consistent with the observation of high similarity among the DNA sequences within each cluster, indicating that individual members of the gene families arose relatively recently. Explanations for this situation have been proposed in a number of other studies on R-genes and involve the evolution of R-gene clusters via duplications and deletions of family members and through gene conversion [10]. The discrepancy of TNL genes in woody plants forming clusters of highly similar genes while TNL genes are generally phylogenetically old, predating the split between gymnosperms and angiosperms, has been noted previously [29]. Studies on the sequenced genomes of grapevine and poplar compared with rice and Arabidopsis indicate that most clustered TNL genes from woody perennials are of a more recent origin than genes from annual plant species [29]. One explanation for this observation is the lower number of generations in woody perennials compared with annuals, which is also held responsible for the generally lower rate of molecular evolution in protein coding genes [28]. Within cluster I of the dendrogram, the rose subclusters include genes from both species (Figure 3), indicating that these copies existed before the species separated. There are only three exceptions to this pattern, among which the gene pair muRdr1B and muRdr1G indicates a very recent duplication event, as the two genes are almost identical. Subclusters IIIa and IIIb harboring genes from Malus (IIIa) and Prunus (IIIb) indicate phylogenetic relationships similar to the Rosa and Fragaria subclusters in that they only include genes from one of the species, which indicates that the genes evolved after Prunus and Malus diverged. The shorter average branch lengths of these clusters compared with all other subclusters indicates that these two groups of genes are the ones that evolved most recently.

\section{Analysis of evolutionary rates}

Enhanced rates of nucleotide diversity are a major factor in the evolutionary dynamics of NB-LRR resistance 
genes [46]. We therefore analyzed both TNL and nonTNL genes across rose, strawberry, apple and peach. In line with the topology of the phylogenetic tree, low values for nucleotide diversity were observed among the Rosoideae (Rosa and Fragaria) compared with the other Rosaceae (Malus and Prunus, Figure 4). However, if we consider the higher rates of evolution among the TNL compared with the non-TNL genes, it is somewhat surprising how closely related the Fragaria TNL genes are to the Rosa TNL genes (Figure 4). This emphasizes the very close phylogenetic relationship between roses and strawberries, which belong to the same subfamily and the same supertribe of the Rosaceae, which is also reflected in the high degree of macrosynteny found in their chromosome structure [42,43]. Although the Rosaceae TNL genes seem to be of relatively recent origin, their nucleotide diversity is more than two-fold higher when compared with the values for the cluster of flanking genes and an arbitrarily chosen gene (Figure 5). This has been observed in several NBS-LRR gene clusters and most likely reflects selective advantages due to higher rates of sequence evolution, which accelerate the evolution of new resistance specificities [46]. For many R-gene clusters, positive selection, indicated by $\mathrm{Ka} / \mathrm{Ks}$ ratios of greater than 1.0, particularly in the LRR regions, has been postulated [3,23,47,48]. We also found increased $\mathrm{Ka} / \mathrm{Ks}$ ratios in the LRR regions of rose $R d r 1$ TNLs. However, in contrast to other studies in which a dramatic increase of $\mathrm{Ka} / \mathrm{Ks}$ values has been observed in LRR regions, we found only moderate increases in the LRR region. This difference might be due to the overall lower rate of sequence evolution observed in perennial plants because of more recent duplications. Alternatively it might be caused by a lower rate of positive selection on the rose $R d r 1$ gene family. There is initial evidence that the Rdr1-TNL cluster includes several resistance genes against black spot and that the evolution of new pathogenic races in black spot is slow due to mostly asexual reproduction and low gene flow [49].

\section{Conclusions}

Our analyses indicate illegitimate recombination, gene conversion, unequal crossing over, indels, point mutations and transposable elements as mechanisms involved in the evolution of the $R d r 1$ locus in rose. It is well documented that similar factors play a role in resistance gene diversity in several annual plant species. Therefore, the diversifying mechanisms associated with the $R d r 1$ locus of the perennial, clonally propagated rose are principally comparable to those of annual plant species, although the Rdr1 TNLs are further characterized by recent duplication.

Analyses of other TNL-type resistance genes in Rosaceae, for example, the recently cloned $M a$ gene from Prunus cerasifera [50], may provide additional information if the pattern of Rdr1-TNL evolution is universal for all TNL genes of perennials or specific to the rose $R d r 1$ alone.

The $R d r 1$ locus is highly conserved within the genus Rosa and somewhat less conserved between Rosa and Fragaria; nevertheless, the synteny is disturbed when compared with Malus and Prunus, possibly due to recombination and chromosome translocation aided by transposable elements.

In the Rosoideae, TNL gene diversification occurred before and after the split of Rosa and Fragaria. A similar phenomenon took place in the Spiraeoideae between Prunus and Malus TNL genes, indicating that most TNL genes in the Rosaceae arose relatively recently.

\section{Methods}

DNA sequence viewing, editing and basic manipulations were performed using Bioedit [51].

\section{Sequencing of the $R$. rugosa BAC clones}

In $R$. rugosa, sequences orthologous to the $R d r 1$ region have been located in four overlapping BAC clones (31C14, 95G17, 78F5 and 35D6) [34]. Sequencing of the four overlapping $R$. rugosa $\mathrm{BAC}$ clones was performed following the procedures described in Terefe-Ayana et al. [35], with a few modifications. Escherichia coli DH10B cells carrying the BAC clones were delivered in stab agar to Cogenics (Cogenics Ltd, Morrisville, NC) for 454 FLX sequencing with $50 \%$ of a full run. The sequences were automatically clipped for adaptor and primer sequences and de novo assembled with Newbler assembler software by Cogenics (Cogenics Ltd, Morrisville, $\mathrm{NC}$ ). Because the BAC clone sequences did not completely assemble into a single contig, subclones were generated from DNA of each BAC clone as described in Terefe-Ayana et al. [35] and Sanger sequenced using commercial sequencing services.

\section{Assembly and gene prediction}

The sequences generated via Sanger sequencing and the first contigs generated using 454 sequencing were assembled with SeqMan (DNASTAR, Madison, WI) and ContigExpress (Invitrogen, La Jolla, CA). Gene prediction and annotation based on the completely assembled sequence was carried out using the gene prediction program FGENESH (http://www.softberry.com). To identify coding sequences in the contig and determine sequence similarity, the whole sequence was fragmented in silico into $1-\mathrm{kb}$ fragments with 200-bp overlaps by the EMBOSS program Splitter (http://emboss.bioinformatics.nl/) and subjected to BLASTn and BLASTx searches [52] against the GenBank database. Domains among the putative protein coding genes were analyzed using Pfam version 23.0 (http://pfam.sanger.ac.uk/) and SMART 6 (http://smart. 
embl-heidelberg.de/). Sequences that were identical to a known gene in GenBank were assigned that gene name.

\section{Origin of strawberry, apple and peach sequences}

The complete genomic sequences of apple, peach and strawberry were downloaded from the Genome Database for Rosaceae (www.rosaceae.org). Regions orthologous to the $R d r 1$ locus and flanking genes were identified using local BLAST searches with the help of Bioedit [51]. Gene predictions for apple, peach and strawberry provided by the respective authors of the genome sequences [53-55] were employed directly, with some additional predictions made using FGENESH (www.softberry.com).

\section{Alignments}

The complete contig sequences of $R$. multiflora [35], $R$. rugosa, Fragaria vesca, Malus $x$ domestica and Prunus persica were aligned using GATA [56] with the default parameters, then compared to determine patterns of gene clusters, the position and orientation of genes and the absence or presence of certain sequence regions.

The predicted $R d r 1$ homologs (TNL homologues) and their flanking genes from each contig of $R$. multiflora, $R$. rugosa, strawberry, peach and apple were aligned using ClustalW with the default options [57]. For the TNL homologues, alignments were carried out for the open reading frame (ORF) and the derived amino acid sequences of the complete gene and separately for each of the TIR, NBS, LRR, exon and intron regions. Alignment of the flanking genes was performed using the complete gene sequences.

\section{Phylogenetic analyses}

For the aligned TNL homologues from the five Rosaceae species, phylogenetic trees were constructed in MEGA5 using the Maximum Likelihood (ML) method based on the Kimura 2 parameter model [58,59]. Phylogenetic analysis of the amino acid sequence was performed using the Jones-Taylor-Thornton (JTT) matrix-based model in MEGA5. Initial trees for the heuristic search were obtained automatically with MEGA 5 . The tree topology was tested via a bootstrap analysis with 500 replicates. The majority rule bootstrap consensus tree was taken to represent the evolutionary history of the taxa analyzed. Branches corresponding to partitions reproduced in less than 50\% bootstrap replicates were collapsed.

\section{Analyses of $\mathrm{Ka} / \mathrm{Ks}$ ratios}

The ratio between the nonsynonymous nucleotide substitutions per nonsynonymous site $(\mathrm{Ka})$ and synonymous nucleotide substitutions per synonymous site (Ks) was evaluated for TIR, NBS and LRR domains separately. The TIR, NBS and LRR domains were determined based on Pfam v23 (http://pfam.sanger.ac.uk/). The amino acid sequences of the different protein domains were aligned in MEGA5 using ClustalW and employed to guide the corresponding cDNA sequence alignment. The resulting cDNA alignments were used to calculate $\mathrm{Ka}$ and Ks with DnaSP [60] following Nei and Gojobori [36]. The selection pattern was characterized by the ratio of Ka to Ks substitution, in which $\mathrm{Ka} / \mathrm{Ks}>1$ indicates positive selection or Darwinian adaptive evolution, $\mathrm{Ka} / \mathrm{Ks}<1$ indicates purifying or stabilizing selection, and $\mathrm{Ka} / \mathrm{Ks}=1$ indicates neutral evolution [61].

\section{Analysis of gene conversion and illegitimate recombination}

The aligned TNL homologues and flanking genes from the five Rosaceae species were analyzed for DNA polymorphisms using $\pi$ applying the Jukes and Cantor correction with DnaSP [60]. This DNA polymorphism analysis indicates the average number of nucleotide substitutions per site between two sequences, and the nucleotide diversity value is the average of all comparisons.

Recombination and sequence exchange between $R d r 1$ homologues from $R$. multiflora and $R$. rugosa was determined with the programs Geneconv [39] and DnaSP [60]. The default parameters were used in both the Geneconv and DnaSP analyses. Events detected with Geneconv were examined and confirmed visually.

\section{Additional files}

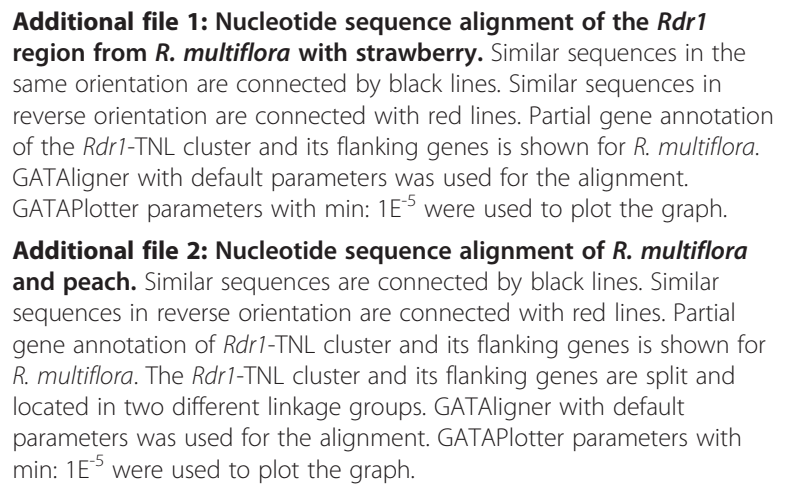

Additional file 2: Nucleotide sequence alignment of $\boldsymbol{R}$. multiflora and peach. Similar sequences are connected by black lines. Similar sequences in reverse orientation are connected with red lines. Partial gene annotation of Rdr1-TNL cluster and its flanking genes is shown for R. multiflora. The Rdr1-TNL cluster and its flanking genes are split and located in two different linkage groups. GATAligner with default parameters was used for the alignment. GATAPlotter parameters with min: $1 \mathrm{E}^{-5}$ were used to plot the graph.

Additional file 3: Nucleotide sequence alignment of $R$. multiflora and apple. Similar sequences are connected by black lines. Similar sequences in reverse orientation are connected with red lines. Partial gene annotation of the Rdr1-TNL cluster and its flanking genes is shown for R. multiflora. The Rdr1-TNL cluster and its flanking genes are split and located on two different linkage groups in apple. GATAligner with default parameters was used for the alignment. GATAPlotter parameters with min: $1 \mathrm{E}^{-5}$ were used to plot the graph.

Additional file 4: Nucleotide sequence alignment of $R$. multiflora and $\boldsymbol{M}$. truncatula. Similar sequences are connected by black lines. Similar sequences in reverse orientation are connected with red lines. Partial gene annotation of the Rdr1-TNL cluster and its flanking genes is shown for R. multiflora. In M. truncatula few TNL genes are shown without having the flanking genes. GATAligner with default parameters 
was used for the alignment. GATAPlotter parameters with min: $1 \mathrm{E}^{-5}$ were used to plot the graph.

\section{Additional file 5: Nucleotide sequence alignment of $R$. multiflora} and $\boldsymbol{A}$. thaliana. Similar sequences are connected by black lines. Similar sequences in reverse orientation are connected with red lines. Partial gene annotation of the Rdr1-TNL cluster and its flanking genes is shown for $R$. multiflora. In A. thaliana few TNL genes are shown without having the flanking genes. GATAligner with default parameters was used for the alignment. GATAPlotter parameters with min: $1 \mathrm{E}^{-5}$ were used to plot the graph.

Additional file 6: Nucleotide diversity ( $\pi$ ) between TNL domains of $R$. multiflora, R. multiflora and R. rugosa, as well as R. multiflora, $R$. rugosa and Fragaria. A significant difference in mean nucleotide diversity can be seen between TIR and NBS/LRR in $R$. multiflora and $R$. rugosa but is masked in the comparison between the three species, $R$. multiflora, R. rugosa and Fragaria.

\section{Competing interests}

The authors declare that they have no competing interests.

\section{Authors' contributions}

TD conceived and designed the experiments. DTA performed BAC subclone preparation, sequencing and assembly. DTA, HK and TD performed sequence analyses, database mining and drafted the manuscript. HK and ML contributed to and edited the manuscript. All authors have read and approved the manuscript.

\section{Acknowledgments}

The study was supported by the German Research Foundation (DFG) grant number DE 511/4-1 and DE 511/4-2.

Received: 18 April 2012 Accepted: 6 August 2012

Published: 20 August 2012

\section{References}

1. Flor HH: Current status of the gene-for-gene concept. Annu Rev Phytopathol 1971, 9:275-296.

2. Dangl JL, Jones JDG: Plant pathogens and integrated defense responses to infection. Nature 2001, 411:826-833.

3. Hulbert SC, Webb CA, Smith SM, Sun Q: Resistance gene complexes: evolution and utilization. Annu Rev Phytopathol 2001, 39:285-312.

4. Baker B, Zambryski P, Staskawicz B, Dinesh-Kumar SP: Signaling in plantmicrobe interactions. Science 1997, 276:726-733.

5. Hammond-Kosack KE, Jones JDG: Plant disease resistance genes. Annu Rev Plant Physiol Plant Mol Biol 1997, 48:575-607.

6. Meyers BC, Dickerman AW, Michelmore RW, Sivaramakrishnan S, Sobral BW, Young ND: Plant disease resistance genes encode members of an ancient and diverse protein family within the nucleotide-binding superfamily. Plant J 1999, 20:317-332.

7. Meyers BC, Kaushik S, Nandety RS: Evolving disease resistance genes. Curr Opin Plant Biol 2005, 8:129-134

8. Jones JDG, Dangl JL: The plant immune system. Nature 2006, 444:323-329.

9. Ellis JG, Lawrence GJ, Luck JE, Dodds PN: Identification of regions in alleles of the flax rust resistance gene $L$ that determine differences in gene-forgene specificity. Plant Cell 1999, 11:495-506.

10. Michelmore RW, Meyers BC: Clusters of resistance genes in plants evolve by divergent selection and a birth-and death process. Genome Res 1998 8:1113-1130.

11. Sudupak MA, Bennetzen JL, Hulbert SH: Unequal exchange and meiotic instability of $R p 1$ region disease resistance genes. Genetics 1993, 133:119-125.

12. Richter TE, Prior TJ, Bennetzen JL, Hulbert SH: New rust resistance specificities associated with recombination in the $R p 1$ complex in maize. Genetics 1995, 141:373-381.

13. Hulbert SH: Structure and evolution of the Rp1 complex conferring rust resistance in maize. Annu Rev Phytopathol 1997, 35:293-310.

14. Sun Q, Collins NC, Ayliffe M, Smith SM, Drake J, Pryor T, Hulbert SH: Recombination between paralogues at the $r p 1$ rust resistance locus in maize. Genetics 2001, 158:423-438.
15. Ramakrishna W, Emberton J, Ogden M, SanMiguel P, Bennetzen JL: Structural analysis of the maize $R p 1$ complex reveals numerous sites and unexpected mechanisms of local rearrangement. Plant Cell 2002, 14:3213-3223.

16. Parniske M, Hammond-Kosack KE, Golstein C, Thomas CM, Jones DA Harrison K, Wulff BBH, Jones JDG: Novel disease resistance specificities result from sequence exchange between tandemly repeated genes at the Cf-4/9 locus of tomato. Cell 1997, 91:821-832.

17. Wulff $B B H$, Thomas CM, Parniske M, Jones JDG: Genetic variation at the tomato Cf-4/Cf-9 locus induced by EMS mutagenesis and intralocus recombination. Genetics 2004, 167:459-470.

18. Seah S, Telleen AC, Williamson VM: Introgressed and endogenous Mi-1 gene clusters in tomato differ by complex rearrangements in flanking sequences and show sequence exchange and diversifying selection among homologues. Theor Appl Genet 2007, 114:1289-1302.

19. Song WY, Pi LY, Wang GL, Gardner J, Holsten T, Ronald PC: Evolution of the rice Xa21 disease resistance gene family. Plant Cell 1997, 9:1279-1287.

20. Meyers BC, Chin DB, Shen KA, Sivaramakrishnan S, Lavelle DO, Zhang Z, Michelmore RW: The major resistance gene cluster in lettuce is highly duplicated and spans several megabases. Plant Cell 1998, 10:1817-1832.

21. Meyers BC, Shen KA, Rohani R, Gaut BS, Michelmore RW: Receptor-like genes in the major resistance locus of lettuce are subject to divergent selection. Plant Cell 1998, 10:1833-1846.

22. Chin DB, Arroyo-Garcia R, Ochoa O, Kesseli RV, Lavelle DO, Michelmore RW: Recombination and spontaneous mutation at the major cluster of resistance genes in lettuce (Lactuca sativa). Genetics 2001, 157:831-849.

23. Kuang H, Woo SS, Meyers BC, Nevo E, Michelmore RW: Multiple genetic processes result in heterogeneous rates of evolution within the major cluster disease resistance genes in lettuce. Plant Cell 2004, 16:2870-2894.

24. Noël L, Moores TL, van der Biezen EA, Parniske M, Daniels MJ, Parker JE, Jones JDG: Pronounced intraspecific haplotype divergence at the RPP5 complex disease resistance locus of Arabidopsis. Plant Cell 1999, 11:2099-2111

25. Dodds PN, Lawrence GJ, Ellis JG: Contrasting modes of evolution acting on the complex $N$ locus for rust resistance in flax. Plant J 2001, 27:439-453.

26. Kuang H, Wei F, Marano MR, Wirtz U, Wang Z, Liu J, Shum WP, Zaborsky J, Tallon L, Rensink W, Lobst S, Zhang P, Tornqvist CE, Tek A, Bamberg J, Helgeson J, Fry W, You F, Luo MC, Jiang J, Buell CR, Baker B: The R1 resistance gene cluster contains three groups of independently evolving, type I $R 1$ homologues and shows substantial structural variation among haplotypes of Solanum demissum. Plant J 2005, 44:37-51.

27. Wicker T, Yahiaoui N, Keller B: Illegitimate recombination is a major evolutionary mechanism for initiating size variation in plant resistance genes. Plant J 2007, 51:631-641.

28. Gaut B, Yang L, Takuno S, Eguiarte LE: The patterns and causes of variation in plant nucleotide substitution rates. Annu Rev Ecol Evol Syst 2011, 42:245-266.

29. Yang S, Zhang X, Yue JX, Tian D, Chen JQ: Recent duplications dominate NBS-encoding gene expansion in two woody species. Mol Genet Genomics 2008, 280:187-198.

30. Horst RK, Cloyd RA: Compendium of rose diseases and pests. Saint Paul: APS Press; 2007.

31. Whitaker VM, Bradeen JM, Debener T, Biber A, Hokanson SC: Rdr3, a novel locus conferring blackspot disease resistance in tetraploid rose: genetic analysis, LRR profiling and SCAR marker development. Theor Appl Genet 2010, 120:573-585.

32. Debener T, Linde M: Exploring complex ornamental genomes: the rose as a model plant. Cr Rev Plant Sci 2009, 28:267-280.

33. Biber A, Kaufmann H, Linde M, Terefe D, Spiller M, Debener T: Microsatellite markers from a BAC contig spanning the Rdr1 locus: a tool for markerassisted selection in roses. Theor Appl Genet 2010, 120:765-773.

34. Kaufmann H, Mattiesch L, Lörz H, Debener T: Construction of a BAC library of Rosa rugosa Thunb and assembly of a contig spanning Rdr1, a gene that confers resistance to blackspot. Mol Gen Genomics 2003, 268:666-674.

35. Terefe-Ayana D, Yasmin A, Le TL, Kaufmann H, Biber A, Kühr A, Linde M, Debener T: Mining disease-resistance genes in roses: functional and molecular characterization of the Rdr1 locus. Front Plant Sci 2011, 2:35. doi:103389/fpls201100035.

36. Nei $M$, Gojobori T: Simple methods for estimating the numbers of synonymous and nonsynonymous nucleotide substitutions. Mol Biol Evol 1986, 3:418-426. 
37. Jukes TH, Cantor CR: Evolution of protein molecules. In Mammalian protein metabolism. Edited by Munro HN. New York: Academic; 1969:21-132.

38. Jiang H, Wang C, Ping L, Tian D, Yang S: Pattern of LRR nucleotide variation in plant resistance genes. Plant Sci 2007, 173:253-261.

39. Sawyer SA: Statistical tests for detecting gene conversion. Mol Biol Evol 1989, 6:526-538.

40. Bennetzen JL: Transposable element contributions to plant gene and genome evolution. Plant Mol Biol 2000, 42:251-269.

41. Terefe D, Debener T: An SSR from the LRR region of the rose Rdr1 gene family is a useful RGA marker for roses and other Rosaceae. Plant Breed 2011, 130:291-293

42. Spiller M, Linde M, Hibrand-Saint Oyant L, Tsai CJ, Byrne DH, Smulders MJM, Foucher $F$, Debener T: Towards a unified genetic map for diploid roses. Theor Appl Genet 2011, 122:489-500.

43. Gar O, Sargent DJ, Tsai CJ, Pleban T, Shalev G, Byrne DH, Zamir D: An autotetraploid linkage map of rose (Rosa hybrida) validated using the strawberry (Fragaria vesca) genome sequence. PLoS One 2011, 6(5): e20463. doi:10.1371/journal.pone.0020463.

44. Jung S, Cestaro A, Troggio M, Main D, Zheng P, Cho I, Folta KM, Sosinski B, Abbott A, Celton JM, Arus P, Shulaev V, Verde I, Morgante M, Rokhsar D, Velasco R, Sargent DJ: Whole genome comparisons of Fragaria, Prunus and Malus reveal different modes of evolution between Rosaceous subfamilies. BMC Genomics 2012, 13:129.

45. David P, Chen NWG, Pedrosa-Harand A, Thareau V, Sévignac M, Cannon SB, Debouck D, Langin T, Geffroy V: A nomadic subtelomeric disease resistance gene cluster in common bean. Plant Physiol 2009, 151(3):1048-1065

46. Yang S, Feng Z, Zhang X, Jiang K, Jin X, Hang Y, Chen J, Tian D: Genomewide investigation on the genetic variations of rice disease resistance genes. Plant Mol Biol 2006, 62:181-193.

47. Chen Q, Han Z, Jiang H, Tian D, Yang S: Strong positive selection drives rapid diversification of R-genes in Arabidopsis relatives. J Mol Evol 2010, 70:137-148

48. Guo YL, Fitz J, Schneeberger K, Ossowski S, Cao J, Weigel D: Genome-wide comparison of nucleotide-binding site-leucine-rich repeat-encoding genes in Arabidopsis. Plant Physiol 2011, 157:757-769.

49. Lühmann AK, Linde M, Debener T: Genetic diversity of Diplocarpon rosae: implications on practical breeding. Acta Hort 2010, 870:157-162

50. Claverie M, Dirlewanger E, Bosselut N, Van Ghelder C, Voisin R, Kleinhentz M, Lafargue B, Abad P, Rosso MN, Chalhoub B, Esmenjaud D: The Ma gene for complete-spectrum resistance to Meloidogyne species in Prunus is a TNL with a huge repeated C-terminal post-LRR region. Plant Physio/ 2011 156:779-792.

51. Hall TA: BioEdit: a user-friendly biological sequence alignment editor and analysis program for Windows 95/98/NT. Nucl Acids Symp 1999, Ser 41:95-98.

52. Altschul SF, Madden TL, Schaffer AA, Zhang J, Zhang Z, Miller W, Lipman DJ: Gapped BLAST and PSI-BLAST: a new generation of protein database search programs. Nucleic Acids Res 1997, 25:3389-3402.

53. Velasco R, Zharkikh A, Affourtit J, Dhingra A, Cestaro A, Kalyanaraman A, Fontana P, Bhatnagar SK, Troggio M, Pruss D, Salvi S, Pindo M, Baldi P, Castelletti S, Cavaiuolo M, Coppola G, Costa F, Cova V, Dal Ri A, Goremykin V, Komjanc M, Longhi S, Magnago P, Malacarne G, Malnoy M, Micheletti D, Moretto M, Perazzo M, Si-Ammour A, Vezzulli S, et al: The genome of the domesticated apple (Malus $x$ domestica Borkh). Nat Genet 2010, 42:833-839.

54. Shulaev V, Sargent DJ, Crowhurst RN, Mockler TC, Folkerts O, Delcher AL, Jaiswal P, Mockaitis K, Liston A, Mane SP, Burns P, Davis TM, Slovin JP, Bassil N, Hellens RP, Evans C, Harkins T, Kodira C, Desany B, Crasta OR, Jensen RV, Allan AC, Michael TP, Setubal JC, Celton JM, Rees DJG, Williams KP, Holt SH, Rojas JJR, Chatterjee M, et al: The genome of woodland strawberry (Fragaria vesca). Nat Genet 2011, 43:109-116.

55. International Peach Genome Initiative (IPGI). http://www.rosaceae.org/peach/ genome.

56. Nix DA, Eisen MB: GATA: a graphic alignment tool for comparative sequence analysis. BMC Bioinformatics 2005, 6:9

57. Thompson JD, Higgins DG, Gibson TJ: CLUSTAL W: improving the sensitivity of progressive multiple sequence alignment through sequence weighting, position-specific gap penalties and weight matrix choice. Nucleic Acids Res 1994, 22:4673-4680.
58. Kimura M: A simple method for estimating evolutionary rate of base substitutions through comparative studies of nucleotide sequences. J Mol Evol 1980, 16:111-120.

59. Tamura K, Peterson D, Peterson N, Stecher G, Nei M, Kumar S: MEGA5: molecular evolutionary genetics analysis using maximum likelihood, evolutionary distance, and maximum parsimony methods. Mol Biol Evol 2011, 28:2731-2739.

60. Librado P, Rozas J: DnaSP v5: A software for comprehensive analysis of DNA polymorphism data. Bioinformatics 2009, 25:1451-1452.

61. Hughes AL: Origin and evolution of Hla class-I pseudogenes. Mol Biol Evol 1995, 12:247-258.

doi:10.1186/1471-2164-13-409

Cite this article as: Terefe-Ayana et al:: Evolution of the Rdr1 TNL-cluster in roses and other Rosaceous species. BMC Genomics 2012 13:409.

\section{Submit your next manuscript to BioMed Central and take full advantage of:}

- Convenient online submission

- Thorough peer review

- No space constraints or color figure charges

- Immediate publication on acceptance

- Inclusion in PubMed, CAS, Scopus and Google Scholar

- Research which is freely available for redistribution 